\title{
DOCENCIA-CREATIVA. Una estrategia de motivación a través de la difusión del conocimiento
}

\author{
Creative-Teaching. A Motivational Strategy Using the Dissemination of Knowledge \\ José Miguel García Ramírez. Universidad de Granada
}

Fecha de recepción: 28 de mayo de 2012 | Fecha de aceptación: 11 de junio de 2012

\section{Resumen}

Docencia-Creativa es un proyecto de innovación docente de la Universidad de Granada con la misión de publicar los trabajos que realiza el alumnado durante el curso académico. Supone una mejora global en el proceso de enseñanza-aprendizaje, porque implica la participación activa y creativa de ambas partes del proceso educativo, docentes y estudiantes.

Palabras clave: Educación | Creatividad | Innovación | Empatía | Motivación

\begin{abstract}
Creative-Teaching is a teaching innovation project of the University of Granada with a mission to publish essays written by the students during the academic year. It represents an overall improvement in the teaching-learning process, because it involves the active and creative participation of both sides of the educational process, professors and students.
\end{abstract}

Keywords: Education | Creativity | Innovation | Empathy | Motivation

La Educación Superior está sometida a un proceso de cambio no sólo a nivel estructural sino también a la necesidad de introducir y de implementar un cambio metodológico (Esteve, 2008; Garcia-Ramirez, 2011). En este sentido, la innovación y la creatividad son necesarias para desarrollar un nuevo modelo pedagógico. El pensamiento creativo aporta aspectos positivos tanto al alumnado como al profesorado, ya que fomenta el emprendimiento, favorece la capacidad de resolución de problemas, facilita el trabajo en equipo, promociona la escucha empática y aumenta la satisfacción personal y laboral. Además, se puede afirmar que el sistema educativo reclama una enseñanza renovada, creativa, interdisciplinar y contextualizada (GarciaRamirez, 2012; Garcia-Ramirez, Garcia-Sempere, Fiorini, 2012).

Por lo tanto, la motivación es una de las claves para la garantía de la calidad en la educación universitaria. La sociedad exige rendimiento y eficacia a la Universidad. A través de las Tecnologías de la Información y la Comunicación, TIC, tanto docentes como estudiantes universitarios construyen y divulgan el conocimiento, dando respuesta a las exigencias sociales. (Esquivias, 2009; Garcia-Ramirez, 2012)

Tomando como precedentes las ideas anteriormente mencionadas, surge el proyecto de innovación docente "Docencia-Creativa. Una estrategia de motivación a través de la difusión del conocimiento" en la Universidad de Granada. 
El proyecto consiste en crear una revista electrónica de investigación con la misión de publicar los trabajos que realice el alumnado durante el curso académico. Por lo tanto, se crea una revista que publica artículos de Educación Superior de carácter científico, académico e interdisciplinar, que sean originales y cumplan una serie de criterios y normas de estilo APA. Estos artículos son procesados por el programa antiplagio Ephorus, después son evaluados de forma anónima por dos miembros del consejo y/o evaluadores/as externos/as. Si las evaluaciones son positivas se publica.

\section{Objetivos}

- Mejorar la docencia, como proceso de enseñanza-aprendizaje

- Incrementar la satisfacción y la motivación en docentes y estudiantes

- Crear un instrumento de evaluación del rendimiento académico

- Divulgar el conocimiento adquirido por el alumnado

\section{Metodología}

Investigación-acción participativa; en la que docentes y estudiantes se implican en un proceso de: transformación de actitudes y comportamiento, aprendizaje social y cooperativo y desarrollo personal de la identidad sociocultural.

\section{Proceso}

- Contexto: Facultad de Ciencias de la Educación y Facultad de Psicología de la Universidad de Granada.

- Participantes: Docentes y estudiantes de todos los grados y másteres de ambas facultades.

- Diagnóstico: La Universidad de Granada, para cumplir con una educación con garantía de la calidad, evalúa anualmente la docencia a través de un cuestionario aprobado por su Consejo de Gobierno. La valoración media en Facultad de Ciencias de la Educación es de aproximadamente 4,00 sobre 5,00. Aunque el rendimiento y la eficacia de la docencia son altos, se puede mejorar motivando a los estudiantes a publicar sus trabajos para divulgarlos en la sociedad. La divulgación del conocimiento adquirido responde a la demanda social; además de ser un vehículo de reconocimiento y eficiencia de una Educación de Excelencia Visible. Por lo tanto, se plantea la creación de ReiDoCrea, Revista electrónica de investigación y Docencia Creativa, partiendo de los recursos existentes.

\section{Previamente al proyecto}

- Durante el mes de mayo de 2011 se crea la revista electrónica.

- Durante los meses de mayo y junio de 2011 el profesorado vinculado al proyecto modifica las guías docentes de las asignaturas.

\section{Durante el proyecto}

- En octubre se explican las normas de publicación.

- A partir de enero se revisan los trabajos.

- En junio de 2012 se publica el volumen 1. 


\section{Cómo se evalúa}

Se aplica la valoración de logro. El logro es la publicación de un trabajo académico; constará de cuatro valoraciones:

- La primera corresponde al profesorado participante; se recoge en la guía docente la cual contiene los criterios de evaluación que aseguran que el alumnado adquiere los conceptos básicos, la capacidad de análisis de la realidad, el sentido crítico y dominio de las claves epistemológicas y teóricas de la materia.

- La segunda valoración de los trabajos es realizada por miembros del consejo o evaluadores externos.

- La tercera valoración de los trabajos es realizada por un comité de iguales, estudiantes del grado participantes en el proyecto.

- La cuarta valoración es una autoevaluación, a través de un relato breve (un máximo de 350 palabras), para explicar su experiencia en esta actividad. Los datos obtenidos se analizan a través del programa Atla.ti.

\section{Qué supone el proyecto para la mejora del aprendizaje}

Este proyecto supone una mejora global en el proceso de enseñanza-aprendizaje, porque implica de una forma participativa y creativa a ambas partes del proceso educativo, docentes y estudiantes. El profesorado orienta y guía al alumnado durante el proceso de aprendizaje de los contenidos necesarios para que adquiera las competencias necesarias de la propia área del conocimiento; pero el profesorado, también, es responsable garantizar la mayor calidad en los trabajos académicos. La publicación de un trabajo académico es un reconocimiento público a los autores; pero este proyecto también conlleva una mejora en eficiencia y satisfacción, porque el estudiante puede sentir que su rendimiento es útil. También forma parte de un proceso de enseñanza-aprendizaje sociocultural consciente de la importancia de la difusión del conocimiento.

\section{Referencias}

Esquivias, MT. (2009). Enseñanza creativa y transdisciplinar para una nueva universidad. Encuentros Multidisciplinares, 31, 43-52.

Esteve, JM. (2008). La formación de profesores en Europa. Hacia un nuevo modelo de formación. Actas del II Congreso anual sobre fracaso escolar. Palma de Mallorca: Govern de les Illes Balears.

Garcia-Ramirez, JM.; Garcia Sempere, P.; Fiorini, M. (2012). Docencia Universitaria y Creatividad. Granada: Editorial Universidad de Granada

Garcia-Ramirez, JM. (2012). La comunicación, clave de excelencia visible en la Educación Superior. Journal for Educators, Teachers and Trainers, 3, 25-36.

Garcia-Ramirez, JM. (2012). Las Tecnologías de la Información y la Comunicación, TIC, en la educación universitaria. Andaluciaeduca, 76, 77.

Garcia-Ramirez, JM. (2011). Una reconsideración de la excelencia visible en la educación superior: la escucha empática. Andaluciaeduca, 66, 84. 